focus on German Studies.

https://journals.uc.edu/index.php/fogs

(ISSN 1076-5697) focus

on German Studies

Issue 27 (2020)

\title{
Die unverfälschte Gemeinschaft. Authentifikationsstrategien einer exklusiven Geselligkeit bei Achim von Arnim und Clemens Brentano
}

\section{Lea Liese}

\section{Universität Basel}

\begin{abstract}
Um 1800, auch vor dem Hintergrund politischer und medientechnischer Entwicklungen (Einführung eines Pressekontrollsystems unter Napoleon, strenge Zensurmaßnahmen durch die Regierung Stein / Hardenberg einerseits, prosperierender Zeitschriftenmarket und Bedürfnis nach Demokratisierung und frei zirkulierendem Wissen andererseits), steuern Achim von Arnim und Clemens Brentano als Dichter der Romantik und politische Akteure des antinapoleonischen Widerstandes über anekdotische Evidenz̧en und Eräählverfahren in Zeitungsmedien und im geselligen Kontext identitätspolitische Ein- und Ausschlusskriterien. Sie nutzen dabei mediale und narrative Authentifikationsstrategien, indem sie verschiedene Nuancen im Spektrum Wahrheit / Fiktion aufgreifen: authentische Berichte, Geschichten ,vom Hörensagen; Erfundenes, historische Quellen und zeitgenössische Debatten. Es wird ein Wissen produziert, das - wiederum in die mündlich-performative Zirkulation eingelassen - effektiv Anschlusskommunikation in anderen gesellschaftlichen Teilsystemen findet und sich dort permanent selbst bestätigt sieht, also Beglaubigung erfährt. In den protokollierten Treffen der, Deutschen Tischgesellschaft" wie auch in den Publikationsorganen (Preußischer Correspondent, Friedensblätter) und literarischen Texten (₹. B. Die Schachtel mit der Friedenspuppe) der politischen Romantik formiert sich somit ein Konzept von Geselligkeit, das eine wahre, ,unverfälschte' Gemeinschaft stiften soll - unter Ausschluss des Weiblichen, Jüdischen, ,Ausländischen ' und ,Philiströsen'.
\end{abstract}

Keywords:

Brentano - Arnim - Deutsche Tischgesellschaft - Anekdote - Geselligkeit

ORCID ID: https://orcid.org/0000-0003-0253-7306

How to cite: Liese, Lea. "Die unverfälschte Gemeinschaft. Authentifikationsstrategien einer exklusiven Geselligkeit bei Achim von Arnim und Clemens Brentano". focus on German Studies 27: Spielformen des Authentischen, no. 27, 2020, pp. 1-25.

DOI: $10.34314 /$ FOGS2020.00002 


\section{Die unverfälschte Gemeinschaft. Authentifikationsstrategien einer exklusiven Geselligkeit bei Achim von Arnim und Clemens Brentano}

Lea Liese

I.

Anekdotische Erzähl- und Schreibweisen, die ab der zweiten Hälfte des 18. Jahrhunderts einen massiven Aufschwung in Literatur, Medien und in geselligen Vereinigungen und zudem eine Politisierung erfahren, entsprechen im besonderen Maße Authentizität erzeugenden narrativen Verfahren.

Die Anekdote gehört zu den kleinen (literarischen) Formen, den genres mineurs', wobei ihr Gattungsname in der Literatur weniger verbrieft ist als in der Historiografie und der Publizistik (Hilzinger, „Anekdote“ 124). Die Gattungsbezeichnung ist abgeleitet von ,anékdota', was im Altgriechischen soviel wie ,Unveröffentlichtes‘ heißt, und geht zurück auf Prokops Historia arcana. Ist die Anekdote heute vor allem dadurch charakterisiert, dass sie eine noch unbekannte, merkwürdige Begebenheit erzählen soll, stellte sie ursprünglich eine unterdrückte, verschwiegene Seite der offiziellen Historiografie dar (Hilzinger, Anekdotisches Eræä̆blen 24). Die Anekdote kann eine Art Gegengeschichte erzählen, auch weil sie nicht primär an der historischen Authentizität interessiert ist. Sie steht somit in Opposition zu den ,grands récits' der Geschichte, indem sie geschichtliche Lücken durch punktuelle Ereignisse ausfüllt und ein öffnendes Moment in den historischen Kontext einführt, so Stephen Greenblatt (19). Dass ihr Kenntnisstand dabei von zufällig erworbenen, einzelnen Fakten geprägt ist, wohingegen systematische Zusammenhänge und tieferes Wissen fehlen, macht sie zu einer leicht zugänglichen Informationsquelle.

Dafür spricht auch das genrebildende Moment des Unveröffentlichten. Der damit verbundene Schwellenstatus zwischen Mündlichkeit und Schriftlichkeit spielt eine besondere Rolle für die bürgerlich geprägte Medienrevolution im 18. Jahrhundert, zum einen aufgrund allgemeiner Alphabetisierungskampagnen und eines stärkeren Medienkonsums im Zuge der 
Aufklärung, zum anderen aufgrund der Zunahme geselliger Verbindungen wie Salons, Lesegesellschaften und akademischen Clubs (Meteling 193-194). So konnte sich das soziopolitische Profil der Anekdote erst nach dem Untergang der Adelskultur schärfen, indem sie unmittelbar auf den Geist der Zeit, also auf die großen politischen und gesellschaftlichen Veränderungen im Zuge der Französischen Revolution reagierte: die Abschaffung der Ständeklausel und der Niedergang des (feudalen) Adels zugunsten einer Emanzipation des Bürgertums, die Ausdifferenzierung in verschiedene Berufe und soziale Gruppen sowie neue Kommunikationsformen und -foren. In den entstehenden Räumen politischer Gegenöffentlichkeit, wo Grenzen des Sagbaren ausgelotet, Meinungs- und Redefreiheit im Kleinen erprobt werden und wo das Politische mit dem Privaten verschränkt ist (Meteling 194195), können Anekdoten den Status einer dynamischen Interferenz zwischen Erzählen, Zuhören, Schreiben und Lesen einnehmen (Hilzinger, Anekdotisches Erzä̈blen 28).

Mit Sonja Hilzinger kann vermutet werden, dass Krisen- und Übergangszeiten - die meist mit einer unsicheren Informationslage einhergehen - die Anekdotenproduktion und verbreitung intensivieren (Anekdotisches Erzählen 75). Dies veranlasst dazu, sie wissenspoetologisch als zeitgeschichtliche, besser: zeitgeistige Dokumente zu deuten, deren mündliche und schriftliche Überlieferungen überdies auf starke Synergieeffekte zwischen den (politischen) Kommunikationsformen in vereinsähnlichen Strukturen einerseits und Zeitungsmedien andererseits hinweisen.

Dieser Medienwechsel (mündlich - schriftlich) ist ein wichtiger Aspekt anekdotischer Inszenierungsstrategien, denn durch ihn kann die Paradoxie ausgeglichen werden, die darin besteht, dass Authentizität einerseits Unmittelbarkeit suggerieren soll, aber dennoch auf mediale Vermittlung angewiesen ist (Bandtel 214-215). Analog zum ,Fiktionspakt ${ }^{6}$, durch den der*die Leser*in mit der fiktiven Welt übereinstimmt, bezeichnet der Authentizitätspakt die Bereitschaft der Rezipient*innen, medialen Erzeugnissen aufgrund von bestimmten narrativen Verfahren Authentizität zuzuschreiben (Weixler 23), sodass die Medialität vergessen werden 
kann. Hierin liegt aber auch die Disposition anekdotischer Schreib- und Erzählweisen, als Instrument des Täuschens und Verstellens instrumentalisiert $\mathrm{zu}$ werden. Vor dem Hintergrund einer immer heterogener werdenden Gesellschaft, in der das Bedürfnis nach freier Geselligkeit, aber zugleich institutionell geregelten sozialen Umgangs- und Kommunikationsformen entsteht, beurteilen zeitgenössische Geselligkeits- und Konversationstheorien den Aufschwung anekdotischen Erzählens somit auch kritisch.

Der spätaufklärerische Aufsatz Über den Umgang mit Menschen (1788) von Adolph Freiherr von Knigge etwa verwehrt sich dagegen, dass vermeintlich intersubjektive Phänomene aus dem gesellschaftlichen Leben prädestiniert seien für eine anekdotische Aufbereitung, beruhten sie doch nicht auf allgemeingültigen Maximen, sondern auf Beobachtungen. Das Erzählen von „kleinen Anekdoten“ (Knigge 25) wird als Negativbeispiel angeführt, wie man soziale Barrieren gerade nicht überwinde, weil die anspielungsreichen Geschichten nur in ihren je eigenen sozialen Wirkkreisen funktionierten. Weil außerhalb dieses Wirkkreises nur Unverständnis zu erwarten sei, sorgten Anekdoten gerade für einen Ausschluss aus der Unterhaltung und seien somit für einen Großteil der Beteiligten „tötend langweilig“ (Knigge 27). Auffällig ist auch Knigges wiederholte Skepsis gegen die soziale Mobilität eines sich kosmopolitisch gebenden Bildungsbürgertums, das Worte zirkulieren lasse wie Handlungswaren, dabei aber nicht die „Kunst“ beherrsche, „sich nach Sitten, Ton und Stimmungen andrer zu fügen“, weil es sich selbst aus Mangel an Erfahrung mit der extrem breiten Auffächerung sozialer Gemeinschaften als absoluter Maßstab setze (Knigge 27). So erscheint bei Knigge ein voraussetzungsreiches, unempathisches Sprechen, das mehr der eigenen Performance und Profilschärfung denn einer gegenseitigen Verständigung und allgemeinen Geselligkeit zu Gute kommt, als Problem der „sogenannten großen Welt“ (332). Ironisch wird zugestanden, dass so ein Verhalten zwar zur höfischen Konversations- und Geselligkeitspraxis gehört haben mag, nun aber überkommen sei, und nicht zur Nachahmung dienen sollte (Knigge 332-334). Denn trotz allen Emanzipationsversuchen scheinen adlige 
Kommunikations- und Geselligkeitsformen auf der imitativen Ebene noch im bürgerlichen Milieu vorzuherrschen, im Glauben, sie seien manierlich. Dass Knigge die so genannte „Anekdotenjagd“ als Adelssignum bzw. -stigma gilt, stellt die Anekdote in einen allgemeinen Zusammenhang mit Verstellung und Falschheit (332-333). Sie scheint hier entgegen ihrer typischen Formkriterien nicht für Authentizität zu bürgen und negativ konnotierten Gesprächsphänomenen wie dem Klatsch strukturell näher zu stehen als literarischen Gattungen. Darüber hinaus gilt das Anekdotische als Schreib- und Erzählweise im Kontext eines unseriösen Sensationsjournalismus, den periodische Publikationsorgane, namentlich „Musen-Almanach(e)“ (Knigge 355) betrieben. So stehen „nichtswürdige Anekdotensammlungen“ in einer Reihe mit „unbedeutende(n) Romane(n), leere(n) Journale(n), platte(n) Schauspiele(n)“ (Knigge 433). Dass der Massengeschmack anekdotische Aufbereitung goutiert, erscheint Knigge als ein Ärgernis seiner Zeit, vor allem, wenn Anekdoten genutzt werden, um zu diskreditieren. Knigge warnt deshalb ausdrücklich davor, Gespräche mit Lästerungen, Spott oder persiflierenden Tönen zu spicken. Anekdoten scheinen also nicht nur in die Nähe eines „leere(n) Geschwätz(es)“ zu rücken, sondern sie entfalten in der Gesellschaft sogar eine asoziale Wirkung (Knigge 50).

In diesem Zusammenhang kritisiert Knigge auch die Übertragungstechnik einer rein auf das Hörensagen beruhenden Erzähltaktik, die er als korrumpierten Nachrichtenaustausch deklariert:

Erzähle nicht leicht Anekdoten, besonders nie solche, die irgendjemand in ein nachteiliges Licht setzen, auf bloßes Hörensagen nach! Sehr oft sind sie gar nicht auf Wahrheit gegründet oder schon durch so viele Hände gegangen, daß sie wenigstens vergrößert, verstümmelt worden, und dadurch eine wesentlich andre Gestalt bekommen haben. Vielfältig kann man dadurch unschuldigen guten Leuten ernstlich schaden und noch öfter sich selber großen Verdruß zuziehn (52). 
Anekdoten erweisen sich hier als Steuerungsmittel öffentlicher (Miss-)Achtung und also öffentlicher Aufmerksamkeit seitens der Rezipient*innen. Somit gewinnt auch der Vergleich von Anekdoten und Schauspielen an Schärfe. Von einer vermeintlichen geselligkeitsfördernden Rolle ist hier nicht im Ansatz die Rede, wohl aber davon, dass eine öffentliche Rufschädigung den Massengeschmack befriedigt.

\section{II.}

Knigges Abhandlung perspektiviert in dieser Hinsicht bereits die exklusive Seite der Anekdote. Hatte sich im Zeichen der Aufklärung ehemals eine assoziativ-offene, tendenziell demokratische Gesellschaftsform gebildet, die Frauen und Juden noch gleichberechtigt einschloss (Matala de Mazza 284), entwickeln sich in der Hoch- und Spätromantik patriotische Männergesellschaften, die über anekdotisches Erzählen in geselliger Runde gemeinschaftliche Ein- und Ausschlusskriterien verhandeln.

Diese Modellierung und Erprobung des Anekdotischen im öffentlichen Raum hängt zusammen mit bestimmten Geselligkeitskonzepten. Knigges Idealvorstellung von Geselligkeit entspricht der einer Nutzengemeinschaft im Gegensatz zu einer autonomen, zweckfreien Geselligkeit, wie sie in der Romantik als Moment der ästhetischen Erfahrung (Kurz 96) postuliert wird (Oesterle 99). Nach Friedrich Schleiermacher befördert diese offene Geselligkeit die Ausbildung einer, elastischeren' Rede- und Schreibweise, die ein Maximum an Anschlusskommunikation bereitstellen sollte (Oesterle 99). Bezogen auf Authentifikationsstrategien bedeutet diese flexible Schaffung von Anschlussmöglichkeiten, „,überall in seiner eigensten Gestalt dazustehen“ und doch zugleich über die ,Fertigkeit` zu verfügen, die ,Oberfläche, die man der Gesellschaft darbietet, nach Erfordern auszudehnen, oder zusammen zu ziehen“" (zit. in Oesterle 99). Das Spiel beider Ebenen stellt nach Günter Oesterle das Potenzial für intellektuelle Netzwerke um 1800 dar, die insbesondere in Berlin 
von einem Wechselspiel der „Allusionspolitik sowohl im Bereich des amüsanten Klatschs wie im Gebiet brisanter auch politischer Nachrichten und Gerüchte“ (103) geprägt sind.

Diese facettenreiche Anspielungskultur findet wiederum kommunikativen Anschluss an die Spannungen zwischen unterschiedlichen gesellschaftlichen und kulturellen Schichten, etwa zwischen den spätaufklärerisch oder romantisch orientierten Netzwerken, zwischen den inklusiven weiblich und jüdisch geprägten Salons und dezidiert antifranzösisch und antisemitisch ausgerichteten Vereinigungen. Wohingegen sich die Salons als „soziale Aktionsräume emanzipatorischen Probehandelns“ verstehen, stellen letztere nach Ethel Matala de Mazza gewissermaßen deren „Zerrbild“ dar (387), das Meinungsfreiheit allerhöchstens in Form von „Gedankenexperimenten mit dem Gestus des Scherzhaften und Spielerischen“ (376) befördere, also mittels einer elastischen Anspielungskultur und flexiblen - performativen - Authentizitätskonzepten, wie im Folgenden am Beispiel der ,Deutschen Tischgesellschaft ${ }^{\star}$ konkretisiert werden soll.

Die ,Deutsche Tischgesellschaft' wird 1811 von Achim von Arnim und dem Staatstheoretiker Adam Müller gegründet und von prominenten Kritikern der Preußischen Reformen, darunter Künstlern (Brentano, August Wilhelm Iffland), Professoren (Schleiermacher, Johann Gottlieb Fichte), Politikern sowie Vertretern des Militärs (z. B. Carl von Clausewitz), besucht. Auch wenn die Versammlungen als unpolitisch ausgegeben werden, üben die Tischgenossen als bedeutende Vertreter der Berliner Eliten einen wesentlichen Einfluss auf die im Wandel begriffene preußische Gesellschaft aus (Nienhaus, „Brentanos Philisterabhandlung“ 245). Hinzu kommt, dass von den finanziellen Beiträgen der Mitglieder ein freiwilliger Soldat mit Waffen ausgestattet und in den Krieg geschickt werden soll.

Das Ziel der Versammlungen ist vorgeblich das Ideal einer reinen, zweckentbundenen Geselligkeit, die sich aber ganz von allein zunächst nicht so recht einstellen will (Nienhaus, „Zur Topik der Tischrede“ 348). Deshalb werden die Mitglieder immer wieder aufgefordert, Anekdotisches vorzutragen; konkret soll ,jeder, der einen unbekannteren Zug vaterländischer 
Treue und Tapferkeit oder überhaupt tüchtiger Gesinnung, oder einen guten ehrbaren Schwank wisse, solchen der Gesellschaft zu allgemeinen Ergötzung“" präsentieren (Brentano, „Vorschläge“ 15).

Hier offenbaren sich zum einen die Authentizität fördernden Synergieeffekte zwischen Versammlungen und Publikationstätigkeiten, Mündlichkeit und Schriftlichkeit, die materialiter in der Einführung eines so bezeichneten „Tagsblattes“ gipfeln (Arnim, „Vorschlag zu einer Deutschen Tisch-Gesellschaft" 6), wie man es eigentlich aus Zeitungen kennt. Schriftliche Medien werden in die Treffen miteinbezogen, entweder zum Zweck der Selbstbeschreibung, wie im Fall protokollführender Dokumente, oder aber als Kommunikationsstifter. Zur Anlage eines „scherzhaften Archivs“ dienen konnten z. B. ein „komische(r) Brief, ein lächerliches Aktenstück, eine sehr lächerliche ZeitungsAnnonce (...), auch Briefe von Wahnsinnigen“ (Brentano, „Vorschläge“ 17).

Zum anderen wird bereits in der Vorrede die Gratwanderung zwischen freier und institutioneller Geselligkeit sowie Scherz und Ernst offenbar: Eine ernste Geschichte soll „mit der Meßerklinge an das Glaß schlagend“ angekündigt werden, hingegen „Scherzzhaftes mit dem Meßerstiele auf den Tisch schlagend“ (Brentano, „Vorschläge“ 15). Weil „Ernst“ und „Scherz“ durch habitualisierte Gesten als solche erkennbar gemacht werden können, ist es ein Leichtes, auch politische Reden, Ressentiment geladene Pamphlete sowie Spott und Lästereien gegen bekannte, aber von den Treffen ausgeschlossene Persönlichkeiten als „Scherz“ auszugeben.

Insbesondere dem Klatsch kommt als inklusive und exklusive Authentifikationsstrategie in dem Zusammenhang eine besondere, weil gruppenspezifische und personengebundene Relevanz zu. Denn Klatsch bedarf einer spezifischen Netzwerkaktualisierung, was bedeutet, dass er selektiv innerhalb eines begrenzten sozialen Netzwerks weitergegeben wird (Bergmann 65, 96). So vollzieht sich der Klatsch hauptsächlich im kleinen Personenkreis und richtet sich gegen bekannte, aber der Gesprächssituation 
ausgeschlossene Dritte. Dabei sind Wahrhaftigkeit und Zeugenschaft und also die Zuverlässigkeit von Informationen Kategorien, die für den Klatsch nicht völlig irrelevant sind, selbst wenn die Teilnehmenden sehr niedrige Standards ansetzen (Adler 73). Auf dem Gebiet der persönlichen Erfahrungen und anekdotischen Evidenzen kann jede ${ }^{*}$ Diskursteilnehmer*in zum*r Expert*in avancieren (Stangneth 133); Klatsch lässt jede*n Anwesende*n mitreden und erklärt jede Aussage für zulässig (Adler 72). Zudem entlastet er von der Verantwortung, ernsthaft für das Gesagte einzustehen, was Diffamierungen aller Art legitimiert. Eine facettenreiche Anspielungskultur, wie sie Oesterle für die Berliner Netzwerke beschreibt, profitiert also strukturell von einer Gesprächsdynamik, die als Klatsch bezeichnet werden kann.

Ironischerweise sind der Klatsch- und Gerüchtekommunikation im hegemonialen Diskurs kulturgeschichtliche Assoziationen mit dem Weiblichen und Jüdischen eingeschrieben. Die Unterstellung von Schwatzhaftigkeit o. Ä. funktioniert, wie wir bereits bei Knigge gesehen haben, selbst als Distinktionsmittel gegen andere soziale oder kulturelle Gesellschaftsschichten, um sie aus dem geselligen Diskurs auszuschließen. Was aber bei Knigge als stilethischer Ratgeber und inklusive Hilfestellung intendiert war, sich in der zunehmend heterogener werdenden Gesellschaft zurecht zu finden, wird im ideologischen Diskurs der politischen Romantik identitätspolitisch instrumentalisiert.

So fordert Arnim schon als Aufnahmebedingung, „daß es ein Mann von Ehre und guten Sitten und in christlicher Religion geboren sey, unter dieser Angemessenheit, daß es kein lederner Philister sey, als welche auf ewige Zeiten daraus verbannt sind“, auch Frauen könnten nicht zugelassen werden („Vorschlag zu einer deutschen Tischgesellschaft“ 5). Alsbald werden auch Brentanos antibürgerliche Abhandlung „Der Philister vor, in und nach der Geschichte“ und Arnims antisemitisches Pamphlet „Über die Kennzeichen des Judenthums“ in einem der Treffen erstmals öffentlich vorgetragen. Diese Tischreden haben eine gruppenkonsolidierende und identitätsstiftende Funktion, weil sie zugleich alles Französische, Jüdische und Weibliche 
ausschließen sollen. In der Betonung des „Antijüdischen“, „Preußischen“ und „Männlichen“ gehen somit Authentifizierungs- mit Exklusionsstrategien einher.

Eine besondere Rolle spielt in diesem Kontext, wie die Forschung herausgestellt hat, die essentialistische Neuakzentuierung der Philistersemantik im Schnittpunkt von Antisemitismus und Nationalbewegung (Bunia et al. 32). Die Philisterthematik steht insbesondere für eine differenzielle Identitäts- und damit zusammenhängend auch Authentizitätsauffassung, die sich mit Selbstwidersprüchen konfrontiert sieht. Ein Selbstwiderspruch, man könnte auch sagen: eine kognitive Dissonanz, besteht darin, dass Philistersymptome immer auch an sich selbst erkannt werden können. Denn „Nichtphiliströsität‘ behauptet im Gegensatz zu der philiströsen, weil sich im Berufsstatus erschöpfenden, festgelegten Identität einen unsicheren Identitätsstatus, der sich durch permanente Selbstbeobachtung vom Anderen abgrenzen muss. Die Wende zum Philiströsen, zu Konfortabilität und Uniformität, etwa bei dem Eintritt in ein bürgerliches Berufsleben, ist im Nichtphiliströsen immer schon markiert (M. Lehmann 117). Deshalb geht die Semantik des Philisterfeindlichen in der politischen Romantik eine Allianz mit dem rassistischen Antisemitismus ein, der im ideologischen Denken eine eindeutige Abgrenzung zu versprechen scheint (Nienhaus, „Brentanos Philisterabhandlung“ 242).

Die Angst vor Unkenntlichkeit manifestiert sich dann im Narrativ des ,Unterwanderns‘. So äußert Arnim eine große Angst, dass „heimliche (weil getaufte, L.L.) Juden“ in die Gesellschaft einziehen könnten („Kennzeichen“ 108). Denn Juden, „listig“ und „still lauernd“ (114), beherrschten die „seltene Kunst sich zu verstekken“ (109). Als „Probe“ wird vorgeschlagen, ihnen „das große Landesunglück von der Jenaer Schlacht“ sowie „vom Tode der Königin (Luise von Preußen, L.L.)“ zu erzählen - der Jude würde alsbald mit seinen ,alten Späße(n)" hervorbrechen und mit Gleichgültigkeit reagieren (125). Ernst hätten sie nur in der „eigenen miserablen Geschichte“ (126). Die vermeintliche „Verkehrtheit der Juden“ (126) wird also an der für die Tischgenossen so wichtigen Ausbalancierung zwischen Ernst und 
Scherz verortet. Zwar seien Juden „närrisch“ und würden „daher zur Unterhaltung einer fröhlichen Tischgesellschaft recht eigentlich dienen,“, doch ihr Scherz träfe meist „an die unrechte Stelle“ (Arnim, „Kennzeichen“126).

Auch in seiner Erzählung Die Versöhnung in der Sommerfrische, die 1811 (also im Gründungsjahr der Tischgesellschaft) veröffentlicht wird, spricht Arnim Juden systematisch Geselligkeit ab. Die Erzählung entsteht wohl unter dem Eindruck einer Auseinandersetzung mit Moritz Itzig, der durch Arnims provokantem Erscheinen im Salon Itzigs Tante Sarah Levy beleidigt wurde und Arnim zum Duell herausforderte. Arnim machte sich über diese Aufforderung lustig, indem er sich - auch im Rahmen der Tischgesellschaft - bei diversen Adligen erkundigte, ob ein Duell mit einem Juden überhaupt standesgemäß sei, was einvernehmend verneint wurde (Moering 1230).

In der Versöbnung wird die Geselligkeit zur Glaubensfrage, weil die Religion darüber entscheidet, wie gesellig sich eine Glaubensgemeinschaft verhält. Vorstellungen von „Volk“ und „Glauben“ werden hier mit Kalkül enggeführt. Wenig überraschend erscheint die christliche Glaubensgemeinschaft als gesellig, sodass schon Juden allein der Geselligkeit wegen zum Christentum konvertiert seien (Versöhnung 557). Doch „es ließe sich mit ihnen kein freudiges deutsches Gemüt teilen, wenn sie auch noch so anständig wären, mitten in der ärgsten Schwelgerei zählten sie ihren Gästen die Bissen in den Mund“" (Versöhnunge 585). Mit dem Jüdischen assoziierte Eigenschaften wie Geldgier werden in dieser Passage als ungesellig und asozial denunziert.

Eine weitere stereotype Attribuierung, die Zuschreibung von Überempfindlichkeit, wird damit erklärt, dass Juden in Gesellschaftskreise eindringen würden, zu denen sie nicht gehörten, und sich infolgedessen immer fremd und verspottet fühlten (Versöhnung 565). Ihre Zerstreuung ,unter fremden Völkern“ wird verglichen mit der Situation der Deutschen unter französischer Besatzung, 
als so viele ihren Volksnamen verändert fanden und sich die neuen Verhältnisse nicht denken konnten, da glaubten sich so viele in der Fremde unter Franzosen verhöhnt, wo jedermann ihnen wohlwollte, einige Minuten Unterredung machten ihnen schlaflose Nächte oder sie fuhren in Beleidigung aus, die niemand sich erklären konnte. (Versöhnung 566)

Die historische und aktuelle Diskriminierung der Juden wird also einerseits auf ein selbstverschuldetes Gefühl reduziert, andererseits durch den schiefen Vergleich, der zudem die die Deutschen als wahre Opfer ausgibt, trivialisiert. Sowohl in Arnims Erzählung als auch in seinem Pamphlet finden sich Narrative - die parasitäre Unterwanderung einer friedlichen Gemeinschaft durch einen Störfaktor, die Verstellungskunst und Falschheit der Juden, die Besinnung auf die christliche Glaubensgemeinschaft als nationale Mission -, die Brentano wenige Jahre später in Die Schachtel mit der Friedenspuppe vervollkommnen und als Leitidee für das mediale Sendungsbewusstsein einer neuen (Herausgeber-)Gemeinschaft der Restaurationszeit umsetzen sollte.

\section{III.}

Mit der Auflösung sozialer Ordnungen - etwa das Ende des Heiligen Römischen Reiches im Jahr 1806 - verlieren auch so genannte ,master narratives` oder ,grands récits` ihre Wirkkraft, die Realität des Sozialen authentisch zu erzählen. An ihre Stelle treten kleine Erzählungen sowie die anekdotische Anverwandlung größerer politischer Kontexte.

Insbesondere im Journalwesen erfährt das Anekdotische, auch im Zuge des napoleonischen Pressekontrollsystems und der preußischen Reformen, eine pragmatischpopuläre Wende. Es kann als Reaktion auf die strengen Zensurmaßnahmen interpretiert werden, die durch die Regierung Stein / Hardenberg durchgesetzt werden und sowohl die (politische) Vereinsbildung als auch Zeitungsmedien massiv einschränken (Lorenz 37-38.). 
Als Arnim im Oktober 1813 die Berliner Tageszeitung Der Preußische Correspondent übernimmt und damit Schleiermacher ablöst, der wegen fortwährender Schwierigkeiten mit der Berliner Zensurbehörde aufgegeben hatte (Knaack 135-136), ist es seine Mission, Spielund Vermittlungsformen zwischen Wahrheit und fiktionaler Aufbereitung im Dienst der unterhaltungs- und informationsbegierigen Leserschaft auszuloten.

„Eine Zeitung muß wiedergeben, was die Zeit ihr darbietet“ („Anzeige“ 424), - in diesem Sinne plädiert Arnim in der Anzeige des Preußischen Correspondenten vom 11. Oktober mit indirektem Verweis auf die strengen Zensurmaßnahmen und die kriegsbedingt schlechte Informationslage für eine Inklusion von Gerüchten, wenn sie sich als politisch nützlich erwiesen („Anzeige“ 424). Daneben forderte er Freunde und Bekannte auf, ihm Anekdotisches zu schicken. Um 1800 rücken tagesaktuelle und zeitgeschichtliche Ereignisse in den Fokus der journalistischen Aufmerksamkeit, weil sie - trotz ihres oft noch ungesicherten Wahrheitsgehalts - authentischer erscheinen als die historische Überlieferung (J. Lehmann 76-77). Die Suche nach der Wahrheit wird nun als prozessual begriffen, was eine serielle Berichterstattung fördert und auch das „Bloß-Wahrscheinliche“ an diskursiver Relevanz gewinnen lässt (Godel 44-45). Denn anekdotisch dargebotenes (Nicht-)Wissen stellt nicht nur einen Ausschnitt, sondern auch einen Möglichkeitsraum dar, der gerade durch seine Unbestimmtheit weitere Aktivitäten anregt - auf der Rezeptions- wie auf der Produktionsebene (Gamper und Mayer 7). Anekdotischen Erzählens ermöglicht es nicht nur, Wahrheiten auszutauschen, sondern Wahrscheinlichkeiten herzustellen, die sich assoziativ reproduzieren, wie Albrecht Koschorke schreibt (36). Alltagskonversation ist erfolgreich, wenn eine erzählte Geschichte immer weitere, ähnliche Anekdoten nach sich zieht (Koschorke 36), systemtheoretisch gesprochen also, wenn Kommunikation anschlussfähig bleibt.

Auf diese Weise entstehen auch Arnims Anekdoten zur Zeitgeschichte, die oft von einer Kriegsberichterstattung nicht klar abzugrenzen sind. In Reaktion auf eine skurrile KriegsAnekdote, die Arnim von Jacob Grimm aus Frankreich zugesandt bekam und die er leicht 
überarbeitet im Preußischen Correspondenten veröffentlichte, erhält er eine irritierte Richtigstellung von dem Soldaten, der die Geschichte selbst ganz anders erlebt hatte. Arnims Anmerkungen in der Ausgabe vom 27. November 1813 zu diesem Fall geben Aufschluss darüber, wie seiner Meinung nach in Krisenzeiten politische Geschichte erzählt werden müsse. Auf den Einwand des Soldaten, dass „(i)n dieser tatenreichen Zeit (...) kleine Abenteuer kein Interesse haben (können), als etwa für die Freunde dessen, der sie bestand“ (zit. in Moering 1336) entgegnet Arnim:

Würden die größeren Begebenheiten vollständig erzählt, so möchten sie den einzelnen Ereignissen das Interesse nehmen, da aber jene eigentlich der Nachwelt erst entschleiert werden, so geben uns diese allein einige Anschauung der Zeit. Die hier berichtigte Anekdote ist von mehreren andern Zeitungen wieder aufgenommen worden, hat unverkennbar ein allgemeines Interesse erweckt und unsere Aufnahme gerechtfertigt. (zit. in Moering 1336)

Zum einen bieten nach Arnim allein die einzelnen Ereignisse einen zeitdiagnostischen Ausschnitt, wenn die größeren Begebenheiten zum gegenwärtigen Zeitpunkt nicht vollständig erzählt werden können. Die lebenswirklichen Episoden des Alltags liefern die authentischeren Zeitdarstellungen, selbst wenn durch „einen weiteren Kreis von Wiedererzählung, (...) in Nebensachen (...) sich manches verändert hat" (zit. in Moering 1336). Zum anderen scheint in Arnims Argumentation vor allem das mediale Interesse eine Publikation zu rechtfertigen, nicht allein Faktentreue.

In diesem Sinne betreiben Arnim und Brentano im Zeitraum zwischen 1810 und 1817 eine Politisierung anekdotischen Erzählens als Authentizitätsstrategie, indem sie vornehmlich Erzählungen verfassen, die den französisch-preußischen Krieg thematisieren und / oder als Fortsetzungsgeschichten in Zeitschriften publiziert werden, teilweise, um mit dem Erlös Invaliden der Freiheitskämpfe zu unterstützen. Bemerkenswert ist, dass Arnim und Brentano ihre in diesen Zeitraum fallenden Erzählungen - nicht ganz gattungstypisch - als „Biographien 
und Anekdoten“ bezeichnen, so Arnim in einem Brief vom 13. Oktober 1814 an Savigny (zit. in Kluge 698). Hier offenbart sich ein enger Bezug der Anekdote zur Biographie als Teil einer alternativen Geschichtsschreibung. Arnim und Brentano entnehmen, denkwürdige‘ Ereignisse aus historischen Quellen, insbesondere Memoiren, verlegen sie in die Gegenwart und reichern den Stoff mithilfe zeitgenössischer Quellen an, wodurch sie ihn politisieren. In der Folge entstehen authentisch scheinende Texte, in denen politische Ereignisse, etwa die Französische Revolution, zum Hintergrund für merkwürdige Lebensereignisse avancieren (Kluge 699).

Dies ist etwa der Fall in Brentanos Erzählung Die Schachtel mit der Friedenspuppe (1815), die um den Jahrestag der Leipziger Schlacht im Herbst 1814 entsteht. Die Erzählung spielt auf einem preußischen Landsitz, als während der Vorbereitung für die Jahresfeier ein Zug Französ*innen aus russischer Gefangenschaft vorbeizieht. In einem Spielzeug der Kinder des Barons - eine Pariser Modepuppe in bunter Schachtel - erkennen sie ein fatales Requisit aus der Vergangenheit und geraten darüber in Streit. Mithilfe des detektivischen Spürsinns des preußischen Barons, der die Französ*innen spontan auf seinem Gut unterbringt, werden mehrere Schicksale aus den Revolutionsjahren zusammengeführt, wobei die sich widersprechenden, lückenhaften und teilweise erlogenen Schilderungen aus der Vergangenheit auf komplizierte Weise miteinander verstrickte Binnengeschichten bilden, die sich erst nach und nach entwirren (Matala de Mazza und Vogl 238): Der bürgerliche Advokat Sanseau und der jüdische Leichengräber Dumoulin hatten sich der Enteignung von Adelsprivilegien, des Erbbetrugs und der Kindesentführung schuldig gemacht und versuchen jetzt, ungestraft und unter falscher Identität, in der postrevolutionären Gesellschaft unterzutauchen, während ihre missbrauchten Opfer, der enteignete Adlige Frenel und seine Frau Antoinette, die als Kind von Dumoulin entführt wurde, von den zurückliegenden Ereignissen traumatisiert, aber in Unkenntnis zurückblieben.

Die den Anspruch des, Unerhörten` erfüllenden Binnengeschichten gehen zum Teil zurück auf eine französische Quelle, nämlich auf die Memoiren des Grafen von Letaneuf aus 
dem 17. Jahrhundert (Kluge 707). Hieraus ergibt sich der anekdotische Anspruch auf biographische Authentizität, so kurios die Ereignisse auch erscheinen mögen. Die Vorgänge werden allerdings in die gegenwärtige Zeit verlegt - die Verbrechen in die Revolutionsjahre und ihre Auflösung in die Restaurationszeit. Entstehungsgeschichtlich fällt die Erzählung mit dem Beginn der Metternichschen Restaurationsbewegung und der Wiedereinsetzung der bourbonischen Monarchie in Frankreich zusammen. Brentano suggeriert in seiner Erzählung das Narrativ, dass in den allgemeinen Revolutions- und Kriegswirren ,natürliche' Genealogien und Ordnungen verwirrt würden. Erst im Zuge der Restaurationsbewegung könnten diese Eingriffe restituiert werden (Matala de Mazza und Vogl 241). Die Erzählung wird demnach als Restaurationsgeschichte interpretiert (Kluge 703).

Dafür spricht auch das Medium, in dem sie publiziert wird: Die von Brentano in Wien mitinitiierte Zeitschrift Friedensblätter, die erstmalig am 30. Mai 1814 erscheint, also am Tag des Pariser Friedensvertrags, berichtet begeistert über den beginnenden Wiener Kongress. Sie wird von der ,Strobelkopf'-Gesellschaft herausgegeben, die den aktuellen politischen Ereignissen der Restauration mindestens wohlwollend gegenübersteht. Brentano bewegt sich hier vor allem im Kreis der Wiener Romantik, zu dem z. B. Anton Passy, Friedrich August von Klinkowström und auch Jacob Grimm gehören. Wenn sie sich auch unpolitisch geben, vertreten die Mitglieder der ,Strobelkopf'-Gesellschaft sehr nationalkonservative und restaurative Anschauungen, die sie mit dem christlichen Glauben verbinden. Viele Akteure besuchten bereits die ,Deutsche Tischgesellschaft ${ }^{6}$ in Berlin oder sind in anderen Männerbünden organisiert. Vor diesem Hintergrund ist die Die Schachtel mit der Friedenspuppe nicht nur eine Journalerzählung, weil sie in einem seriellen Publikationsmedium erscheint, sondern sie ist politisch, weil sich zwischen Erzählung und Publikationsmedium programmatische Bezüge identifizieren lassen.

Zunächst ist auffällig, dass Zeitungsmedien in der Erzählung explizit erwähnt bzw. in den Handlungsverlauf integriert werden. So dürstet es den preußischen Baron, nachdem er 
aus dem Krieg zurückgekehrt ist, nach einer heimischen Zeitung, deren „liebkosende“ Lektüre symptomatisch für sein quasi-libidinöses Verhältnis zum „Vaterland“ steht:

(...) und der Baron hieb eine Birke um, den Block damit zu lüften, als die Baronin mit der Zeitung den Hügel heraufkam. Er warf sein Beil nieder und durchlief die Blätter mit der Begierde, die ihm, der lange von dem Vaterlande im Kriegstreiben getrennt, sehr natürlich war. Alles ist an den Blättern, die ruhig das Forum und den Gemüsemarkt des täglichen Lebens ausstellen, unter solchen Umständen interessant, ja selbst die ewig wiederkehrenden Namen der Auktionskommissaire, Buchhändler, und Schenkwirte. Die Baronin folgte seinen Blicken; die Ungeduld, mit welcher er las und alles Vaterländische liebzukosen schien, tat ihr selbst wohl. (Friedenspuppe 318f.)

Was hier für den Baron von Relevanz ist, sind nicht etwa globalpolitische Zusammenhänge, sondern das, was ihm ein Heimatgefühl stiftet: das Kleine, das Alltägliche, das Lokale. Die vermeintlich harmlose Berichterstattung über wiederkehrende Trivialitäten aus dem täglichen Leben ist aber nur auf den ersten Blick eine unpolitische. Dass der Baron mit dieser erzählten Lebenswirklichkeit „Vaterländisches“ identifiziert, überhaupt der gleich zweifache Verweis auf das „Vaterland“ in dieser doch so nebensächlich anmutenden, kurzen Passage, offenbart den Chauvinismus der Befreiungskämpfer, deren Patriotismus sich nun nach dem Sieg über Napoleon und die französische Vorherrschaft in einem offenen Nationalismus Bahn brechen kann. Schließlich erweisen sich die Lokalnachrichten, die hier eingestreut werden, als gezielte Vorbereitung für die eigentlich handlungslogisch entscheidende Information, die der Baron aus seiner Zeitungslektüre ziehen soll: Brentano positioniert im Folgenden den expliziten Hinweis auf einen real existierenden Zeitungsbeitrag, nämlich auf den Aufruf zur Feier des Jahrestages der Schlacht bei Leipzig in der Spenerschen Zeitung vom 11. Oktober 1814. In der Erzählung heißt es: „,Gut! Das muß geschehen, sagte der Baron, und zwar hier auf der Stelle.' Die Baronin fragte, was er meine, und er las ihr aus der Vossischen Zeitung die Aufforderung eines deutschen Patrioten vor, den 18. Oktober, 
den Jahrestag der Leipziger Schlacht, mit Freudenfeuern auf allen Anhöhen zu feiern“ (Friedenspuppe 319).

Die Feierlichkeiten auf der Handlungsebene werden also ebenso durch einen Zeitungsaufruf motiviert wie auf der außerliterarischen, allerdings verlegt Brentano den realen Beitrag aus der Spenerschen in die Vossische Zeitung (Kluge 724). Der besagte Aufruf in der Spenerschen Zeitung bezieht sich dann auch noch ausdrücklich auf einen Vorschlag des ultranationalistischen, antisemitischen Schriftstellers Ernst Moritz Arndt, dessen Werke zu einem großen Teil der Mobilisierung gegen Napoleon sowie den Befreiungskriegen gewidmet sind.

Aber nicht nur die Motivation zu einer Friedensfeier hat in der medialen Stimmungslage einen stimmungsvollen Referenzort. Brentano hat sich auch zeitgenössischer Zeitungs- und Augenzeugenberichte bedient, um die Festaktivitäten authentisch darzustellen. Zum Beispiel entnimmt er die Erscheinung eines Meteors am Nachthimmel, der als „Feuerball“ die allegorische Ergänzung zu den Freudenfeuern der Deutschen bildet, der medialen Berichterstattung (Kluge 748). So schreibt die Spenersche Zeitung vom 20. Oktober 1814: „Ein noch helleres Feuer leuchtete inzwischen als glückliches Zeichen vom Himmel herunter, und zog eine Viertelstunde lang sichtbar als Feuerkugel vorüber“ (zit. in Kluge 743). Und die Ausgabe vom 3. November präsentiert den Augenzeugenbericht eines Pfarrers: „Auch schien der Himmel sich dieses ländlichen Schauspiels zu freuen, denn es durchschimmerten späterhin zwei leuchtende Meteore sein tiefes Lasur! - Ja, wir sind Teutsche, und wollen, das schwuren wir Angesichts des ganzen zuschauenden Himmels, als Teutsche leben und sterben (...)“ (zit. in Kluge 747). In Brentanos Erzählung wiederum heißt es:

Gegen zehn Uhr erschien (...) eine Feuerkugel, (...) und nun erhoben die Freudenfeuer der Deutschen, welche von der himmlischen Erscheinung zu verlöschen schienen, um so sehnsüchtiger und dankender ihre Flammen zur Nacht empor. Es war, als habe der Himmel sagen wollen: ,Ihr leuchtet mit Freudentränen, wenn ich aber mit meinem 
Lichte euch erleuchte und die Nacht euch nehme, so sinken eure Flammen ein. Seht, mir gefällt euer kindisches Spiel, und ich gönne euch die heilige Nacht; aber wie ihr alle meine Feuer gesehen habt, unter einander aber nur jeder das seine, oder das der nächsten Nachbarn, so gedenket, daß nur das Licht von oben ein einigendes ist, (...). (Friedenspuppe 350)

Brentanos pathetische Inszenierung kosmischer Einheit und die Versinnbildlichung göttlichen Wohlwollens durch die Gestirne ist keine rein romantische Fiktion, sondern schließt an medial geförderte, zeitgenössische Vorstellungsbilder an. Weil unterschiedliche mediale Formen kombiniert werden, wird der Authentizitätsfaktor nicht durch die allegorische Überhöhung beeinträchtigt. Die Wechselwirkungen zwischen literarischem Text und Zeitungsmeldung stellen sich so als Teil einer Authentifikationsstrategie dar.

Die Festpassage steht zudem in deutlichem Kontrast zu der Darstellung eines jakobinischen Festes, wie sie Frenel aus Erzählungen rekonstruiert:

Zu dem Feste hatte der junge Sanseau mehrere Gedichte verfertigt; er bestürmte seine Geliebte, die Mademoiselle Montpreville, die Rolle der Gleichheit zu übernehmen, (...). Der Gleichheitsrock wurde geschneidert und angezogen. (...) Sie waren alle frei und gleich, obschon sie eine ziemlich garstige Gleichheit vorstellte, denn sie war häßlich; (...) so war sie doch auf der einen Schulter etwas zu uneben, und die Pariser Witzlinge bemerkten, als sie in dem Tempel der Vernunft spazierte, daß sie (...) eine Achselträgerin, daß sie noch nicht ganz gleich sei. (...) Da erschien auf einer kleinen Bühne der junge Sanseau als der Patriotismus, der zum Kampfe ziehen wollte; er sah die Noblesse, Mademoiselle Montpreville, unter einem Stammbaum mit vielen Wappen schlummern, der, vom Blitze getroffen, niederzustürzen und sie zu zerschmettern drohte; er bedauerte ihre Gefahr, er wollte sie wecken. (...) Sie umarmte den Stammbaum, da führte sie der Patriotismus in den Tempel der Freiheit; diese riet ihnen, 
sich eine Hütte aus dem alten Stammbaum zu bauen, und kleidete die Noblesse als Egalité ein, (...). (Friedenspuppe 337-338).

Hier wird religiös motivierte Kritik an den atheistischen Kultfesten der Französischen Revolution und deren ikonoklastischen Praktiken geübt: Die personifizierte Noblesse wird durch den Patriotismus verführt und als Egalité eingekleidet, oder vielmehr vorgeführt und verkleidet, denn die Gleichheit ist eine entstellte, "garstig“ und „häßlich“. Durch diese suggestive Gegenüberstellung sollen der deutsche Patriotismus und die Gleichheit im Glauben an Gott als ,wahr' legitimiert, während der jakobinische Patriotismus und die revolutionäre Egalité als denaturiert delegitimiert werden.

Entscheidender aber als diese antifranzösischen Klänge ist, dass hier das Narrativ vom Sieg über die Französische Revolution auch ein antisemitisches ist, wie Matala de Mazza und Vogl richtig erkennen (248). Am Ende erscheint zwar die Jahresfeier der Leipziger Schlacht als Fest der Buße und Versöhnung, durch das die Menschen (wieder) im Bund mit Gott stehen. Aber dem harmonischen Menschenbund, in dem schließlich sogar die französischen und deutschen Protagonist*innen miteinander versöhnt sind, wird bei Brentano mit dem bestechlichen Leichengräber Dumoulin die antisemitische Karikatur des Juden als ultimatives Feindbild entgegengesetzt.

Dumoulin bleibt fernab des geselligen Treibens und also außerhalb der Gemeinschaft. Nicht mit Freude, sondern „mit großem Schmerz“ nimmt er an den Feierlichkeiten teil und richtet sich schließlich selbst (, unter den vielen Freudenschüssen, die rings gefallen sind, war auch der, der seinem Leben ein Ende machte“, Friedenspuppe 351), weil seine Taten offenbar wurden und er Bestrafung und Enteignung fürchtet. Dumoulin stirbt also während des Festes „wie ein Feind der Freiheit und des Friedens“ (Friedenspuppe 351). Im Gegensatz zu dem Franzosen Sanseau bereut er seine Taten nicht; er scheint immun gegen den Aufruf zur Beichte und Versöhnung am Tag des zum Jüngsten Gerichts allegorisierten Friedensfestes. 
Die Rhetorik der Erzählung Die Schachtel mit der Friedenspuppe findet somit auf der einen Seite Anschluss an die Rhetoriken der Zeitungen, Stammtische und Männerbünde, die darin bestehen, ein organisches, eindeutig identifizierbares Feindbild gegenüber der deutschen Sprache und Kultur heraufzubeschwören. Brentanos Narrativ findet auf der anderen Seite auch realpolitisch Rückbindung, weil im Zuge des Wiener Kongresses, über den die Friedensblätter ihr Lesepublikum ja ausführlich unterrichteten, die unter französischer Herrschaft beschlossenen Maßnahmen wieder zurückgenommen werden, darunter auch das Bürgerrecht für Juden (Matala de Mazza und Vogl 248).

Man kann hier von einer kognitiven Dissonanz sprechen, die darin besteht, einerseits die Modernisierung des preußischen Staates und die demokratische Teilhabe der Gesellschaftsmitglieder an politischen Prozessen $\mathrm{zu}$ fordern, sich andererseits gegen demokratische Rechte auch für vermeintliche Minoritäten zu wehren. Diese kognitive Dissonanz findet ihre identitätspolitische Entsprechung in der essentialistischen Neusemantisierung der Philisterthematik durch antisemitische Allianzbildungen. Flexible Authentifizierungsstrategien lassen Selbstwidersprüche dieser Art aushalten. Der Hass gegen Juden, Frauen, Franzosen und Philister entfaltet sich auf dem schmalen Grat zwischen Ernst und Scherz, kollektiver Identitätsstiftung nach innen und ideologischer Abschottung nach außen. Das Spiel mit der Ununterscheidbarkeit bezieht seine Kraft aus der Performanz des Erzählens.

IV.

Authentisches Erzählen in der Spätromantik steht unter dem Zeichen der Restaurationsbewegung und entfaltet sich in den Kommunikationsformen (Anekdote) und foren (Zeitungen, gesellige Treffen), die seit ihrer Prägung in der französischen Aufklärung im postrevolutionären und antinapoleonischen Deutschland eine politische Wende erfahren haben, an den Schnittstellen von Mündlichkeit und Schriftlichkeit sowie Demokratisierung 
und Nationalismus. Die Gattung der Anekdote ist dabei als Medium politischer Narrative besonders wirksam, weil patriotische Schriftsteller der Romantik sie sowohl als Geselligkeit erzeugendes als auch als zensurtaugliches Agitationsmittel erkennen. Die Anekdote erreicht durch die ihr inhärenten gattungstypischen Merkmale (Oralität, Verdichtung, Anschlussfähigkeit) und durch ihre Medialisierung sowohl historisch tradierter Narrative als auch zeitgenössischer Debatten einen besonderen Authentizitätsfaktor. Die medialen und narrativen Kolportagestrategien, die kleine Erzählformen vorführen, reproduzieren das (oftmals) reaktionäre Stimmungsbild zeitgenössischer Publikationsmedien und perspektivieren die erwartbare Stimmung einer Leserschaft, die sich beinahe zeitgleich im Beobachtungsmodus der aktuellsten politischen Ereignisse befindet. Somit wird nicht nur binnenliterarisch eine fiktive Gemeinschaft unter Ausschluss des ,Fremden“ heraufbeschworen - etwa wie in Brentanos Erzählung Die Schachtel mit der Friedenspuppe sondern auch in den sendungsstarken politischen Teilöffentlichkeiten: „Die deutsche Tischgesellschaft // Bewahrt sich drum mit gleicher Kraft // Vor den Philistern und vor Juden, // Damit sie wächst in allem Guten“ (Arnim, „Kennzeichen“ 371). 


\section{$\underline{\text { Literaturverzeichnis }}$}

Adler, Jonathan E. „Gossip and Truthfulness.” Cultures of Lying. Theories and Practice of Lying in Society, Literature, and Film, hrsg. von Jochen Mecke, Galda + Wilch Verlag, 2007, S. 6978.

Von Arnim, Achim. Die Versöhnung in der Sommerfrische, hrsg. von Renate Moering, Deutscher Klassiker Verlag, 1990.

---. Anekdoten zur Zeitgeschichte, hrsg. von Renate Moering, Deutscher Klassiker Verlag, 1990.

---. „Anzeige“. Schriften, hrsg. von Renate Moering, Deutscher Klassiker Verlag, 1992.

---. „Über die Kennzeichen des Judentums.“ Stefan Nienhaus, Niemeyer, 2008.

---. „Vorschlag zu einer deutschen Tisch-Gesellschaft.“ Texte der deutschen Tischgesellschaft, hrsg. von Stefan Nienhaus, Niemeyer, 2008.

Bandtel, Matthias. „Authentizität in der politischen Kommunikation. Mediale Inszenierungsstrategien und authentifizierende Selbstdarstellungspraktiken politischer Akteure." Authentisches Eræählen. Produktion, Narration, Rezeption, hrsg. von Antonius Weixler, De Gruyter, 2012, S. 213-236.

Bergmann, Jörg R. Klatsch. Zur Sozialform der diskreten Indiskretion, De Gruyter, 1987.

Brentano, Clemens. Die Schachtel mit der Friedenspuppe, hrsg. von Gerhard Kluge, W. Kohlhammer, 1987.

---. „Vorschläge.“ hrsg. von Stefan Nienhaus, Niemeyer, 2008.

Bunia, Remigius et al. „Elemente einer Literatur- und Kulturgeschichte des Philisters. Einleitung." Philister. Problemgeschichte einer Sozialfigur der neueren deutschen Literatur, hrsg. von Remigius Bunia et al., De Gruyter, 2011, S. 13-51.

Gamper, Michael, und Ruth Mayer, Hrsg. „Erzählen, Wissen und kleine Formen. Eine Einleitung.“ Kurz \& Knapp. Zur Mediengeschichte kleiner Formen, Transcript, 2017, S. 7-22. 
Godel, Rainer. „Literatur und Nicht-Wissen im Umbruch, 1730-1810.“ Literatur und NichtWissen. Historische Konstellationen 1730-1930, hrsg. von Michael Bies und Michael Gamper, Diaphanes, 2012, S. 39-58.

Greenblatt, Stephen. „Introduction: Joel Fineman's Will.“ The Subjectivity Effect in Western Literary Tradition: Essays Towards the Release of Shakespeare's Will, hrsg. von Joel Fineman, Cambridge, 1991, S. ix-xix.

Hilzinger, Sonja. „Anekdote.“ Handbuch der literarischen Gattungen, hrsg. v. Dieter Lamping, Kröner, 2009, S. 12-15.

---. Anekdotisches Erzäblen im Zeitalter der Aufklärung. Zum Struktur- und Funktionswandel der Gattung Anekdote in Historiographie, Publizistik und Literatur des 18. Jabrbunderts. M und P Verlag, 1997.

Knaack, Jürgen. „Achim von Arnim und der ,Preußische Correspondent‘. Eine letzte großstädtische Aktivität vor dem Umzug nach Wiepersdorf.“ Universelle Entwürfe Integration - Rückzug. Arnims Berliner Zeit (1809-1814): Wiepersdorfer Kolloquium der Internationalen Arnim-Gesellschaft, hrsg. von Ulfert Ricklefs, Niemeyer 2000.

Knigge, Adolf Freiherr Knigge. Ueber den Umgang mit Menschen, hrsg. von Michael Rüppel, Wallstein, 2010.

Kurz, Gerhard. „Das Ganze und das Teil. Zur Bedeutung der Geselligkeit in der ästhetischen Diskussion um 1800.“ Kunst und Geschichte im Zeitalter Hegels, hrsg. von Christoph Jamme, Meiner, 1996, S. 91-113.

Lehmann, Johannes F. „Was der Fall war: Zum Verhältnis von Fallgeschichte und Vorgeschichte am Beispiel von Lenz' Erzählung ,Zerbin'“"Was der Fall ist. Casus und Lapsus, hrsg. von Mülder-Bach, Inka, und Michael Ott, Wilhelm Fink, 2014, S. 73-87.

Lehmann, Maren. „Philiströse Differenz. Die Form des Individuums.“ Bunia et al., De Gruyter, 2011, S. 101-120.

Lorenz, Dagmar. Journalismus. Metzler, 2009. 
Matala de Mazza, Etel. „Sozietäten (Christlich-Deutsche Tischgesellschaft).“ Kleist-Handbuch, hrsg. von Ingo Breuer, Metzler, 2009, S. 283-285.

Matala de Mazza, Etel, und Joseph Vogl. „Poesie und Niedertracht. Über Brentanos Restaurationsgeschichte.“ Die Lesbarkeit der Romantik. Material, Medium, Diskurs, hrsg. von Erich Kleinschmidt, De Gruyter, 2009, S. 235-250.

Meteling, Arno. „Verschwörungstheorien. Zum Imaginären des Verdachts.“ Die Unsichtbarkeit des Politischen. Theorie und Geschichte medialer Latenz, hrsg. von Lutz Ellrich et al., Transcript: Bielefeld 2009, S. 179-212.

Nienhaus, Stefan. „Brentanos Philisterabhandlung und ihre Kommentierung im Rahmen der historisch-kritischen Edition." Philister. Problemgeschichte einer Sozialfigur der neueren deutschen Literatur, hrsg. von Remigius Bunia et al., De Gruyter, 2011, S. 241-251.

--- Philister. Problemgeschichte einer Sozialfigur der neueren deutschen Literatur, hrsg. von Remigius Bunia et al., De Gruyter, 2011. „Zur Topik der Tischrede: ,Verehrte Tischgenossen“، Topik und Rhetorik. Ein interdisziplinäres Symposium, hrsg. von Thomas Schirren und Gert Ueding, Niemeyer, 2000, S. 345-354.

Oesterle, Günter. „Diabolik und Diplomatie. Freundschaftsnetzwerke in Berlin um 1800.“ Strong ties / Weak ties. Freundschaftssemantik und Netzwerktheorie, hrsg. von Natalie Binczek und Georg Stanitzek, Winter, 2010, S. 93-110.

Stangneth, Bettina. Lügen lesen. Rowohlt, 2017.

Weixler, Antonius. „Authentisches erzählen - authentisches Erzählen. Über Authentizität als Zuschreibungsphänomen und Pakt." Authentisches Erzäblen. Produktion, Narration, Rezeption, hrsg. von Antonius Weixler, De Gruyter, 2012, S. 1-32. 